\title{
Evaluating the effect of tolerances on the functional requirements of assemblies
}

\author{
Mehdi Tlija $^{1}$, Borhen Louhichi ${ }^{1,2, a}$ And Abdelmajid BenAmara ${ }^{1}$ \\ 1 LGM, ENIM, University of Monastir, Av. Ibn Eljazzar, 5019 Monastir, Tunisia \\ 2 LIPPS, ÉTS, 1100 Notre-Dame Ouest, Montréal, H3C1K3 Québec, Canada
}

Received 12 July 2012, Accepted 26 March 2013

\begin{abstract}
In order to improve digital mock-up, a tolerancing phase should be integrated in the geometric models. However, in CAD software, tolerances are represented by annotations, which are neglected as well as the tolerance impact. Thus, the system malfunction is generated. For these reasons, in this paper a tolerancing phase is integrated in the numerical model to form a realistic model, where worst case configurations of assemblies are determined from the tolerances assigned to the nominal model. The proposed model incorporates tolerances on CAD models in the case of planar face, cylindrical face and planar face with non quadratic loop. In addition, the model ability to respect the maximum material condition (MMC) and the requirement of datum priority order in the CAD models is shown. Finally, functional requirement of a linear guide mechanism is inspected by using the proposed model.
\end{abstract}

Key words: CAD integration / tolerancing / modelling / realistic assembly / MMC

\section{Introduction}

In the Digital Mock-Up (DMU), the simulation of the mechanical deformations and the prediction of the functional requirements represent two main phases of the product design. The results of those calculations depend on adopted CAD model. In the DMU, the part and the assembly are represented by their nominal dimensions. In fact, tolerances are defined as annotations and are neglected in the geometric model. Consequently, neglecting tolerance impacts causes system malfunctions at mountain process and during the operating phase. In the current Product Life cycle Management (PLM), the detection of those tolerance impacts is made only after the product realization [1]. The correction of these errors at this stage requires additional costs [2]. Hence, the prediction of these errors represents an industrial need and a financial gain, and can be performed by using a realistic model (Fig. 1).

Thus, the main objective of this paper is to integrate the tolerancing in the CAD model by determining worst case configurations of components and assemblies: realistic models. A model taking into account dimensional tolerances has been developed in our previous work [3]. In this paper, worst case configurations are modelled using the geometrical tolerances. First, a part of extremely rich literature is presented. Then, steps of the proposed

${ }^{a}$ Corresponding author: Borhen.Louhichi@etsmtl.ca method are defined. The proposed algorithm is ameliorated in order to consider the MMC (maximum material condition) and the requirement of datum priority order in the CAD model. Finally, tolerance analysis of linear guide mechanism is realized by using the proposed method.

\section{A case study}

\subsection{Modelling of tolerance zones}

The improvement of tolerancing leads to an industrial success. The modelling and the representation of tolerance zones lead to the establishment of many structural concepts. The tolerance integration in the CAD model requires a mathematical representation of the tolerance zones. The tolerancing modelling had been intensively investigated by researches such as: the envelope zone [4], the variational, the parametric, the vectorial [5], the TTRS (topologically and technologically related surfaces) [6] and unified Jacobean - torsor models [7].

The TTRS approach leads to the tolerancing with domains method. The tolerancing with domains method is based on the concept of the SDT (Small Displacement Torsor). This concept neglects the deviation form and simplifies the three-dimensional problems by linearization of the matrix expressing the displacements of an object [8]. This approach is a tool of analysis and synthesis of the geometrical tolerances [9]. 


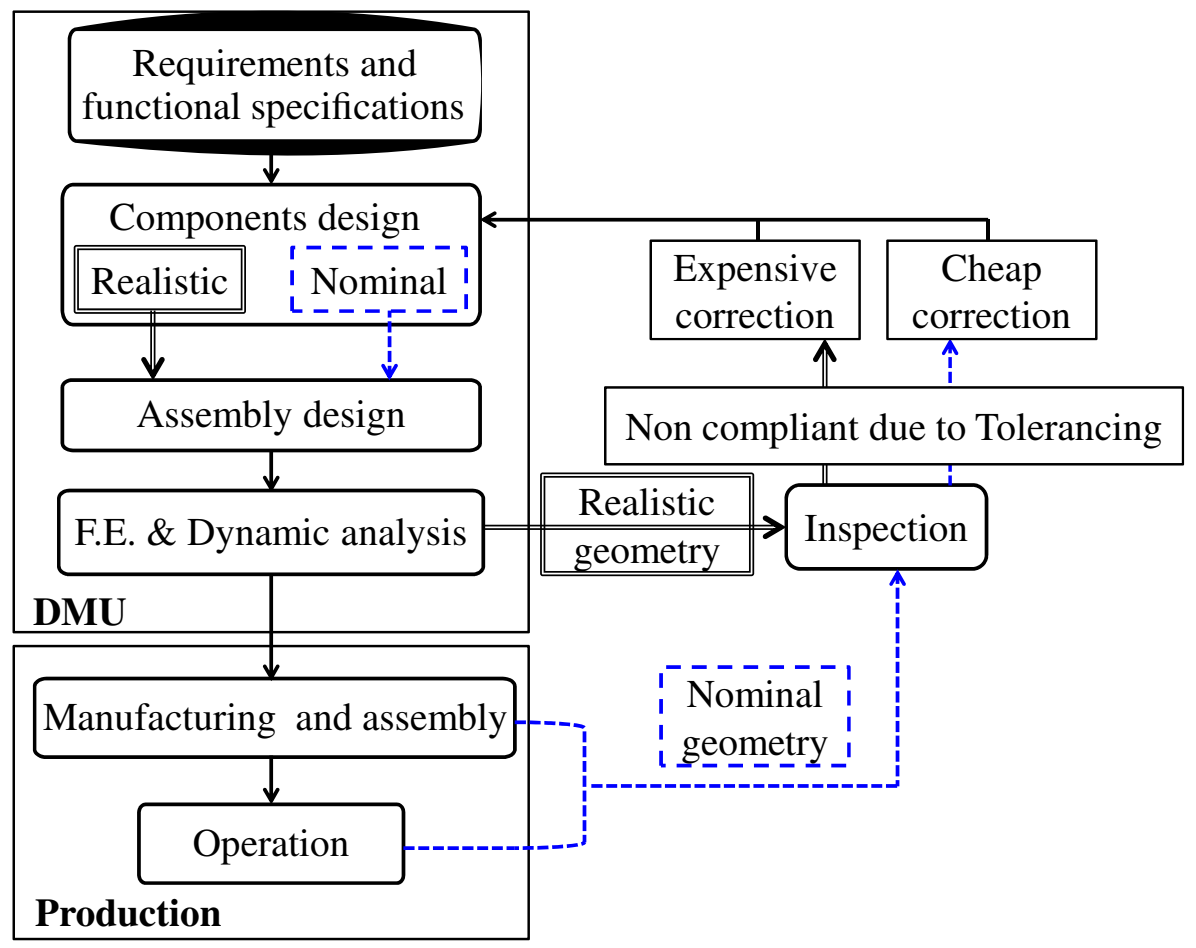

------ Current Product Lifecycle Management

ב Product Lifecycle Management (incorporating Tolerancing in DMU)

Fig. 1. Correction of design defects caused by tolerances in an early phase of product life cycle management.

In addition, the variability of manufacturing processes generates tolerancing problems. Then, research works have focused on the influence of processes dispersion and errors of machine tools on manufacturing tolerances. Based on a graphical representation (matrix), the dispersion method (the $\Delta l$ method) enables the establishment of unidirectional dimension chains. This method had been improved to achieve three-dimensional chains of manufacturing dimensions [10]. Tichadou et al. proposed a model of three-dimensional dispersion of manufacturing and developed a chart representation. This representation allows expressing the geometrical manufacturing conditions of the process plan as a torsor chain. Indeed, each geometrical manufacturing condition is defined as constraints on torsor components [11]. Vignat et al. proposed a MMP (Model of Manufacturing Part) to perform three-dimensional manufacturing tolerances synthesis. The SDT tool is used to define the surface displacements which are deduced from the result of the tolerance analysis. The defects generated by the process (positioning and manufacturing) are identified and classified. Then, the MMP is used to perform the analysis of functional specifications according to ISO manufacturing tolerances [12]. Benea et al. developed a kinematic modelling of manufacturing defects that allows simulating manufacturing steps of the part. This model uses the graph tool and the Jacobean matrices for representing the machining sequences and the manufacturing defects respectively [13].

\subsection{Modelling of geometric deviations in CAD model}

The prediction of the geometrical deviations between the real and the nominal assembly has interested many scientific researches. In this paper, some of those researches are presented.

Chang and Gossard propose an assembly modelling of deformable and non ideal parts during processes of welding and measurement [14]. The graph tool is used to identify relations between assembly features, displacements, position and applied loads. Besides, deduced relationships are translated into equations while respecting the force continuity and the geometric compatibility. Solutions of these equations represent the deviations propagation during the process. Then, variations in the resulting assembly are determined. This method is applied on particular cases and it has not been generalized or automated.

Ballu et al. propose a solution for the tolerancing integration in the numerical model by using the parametric modelling of only functional surfaces [15]. The model is integrated in CATIA V5 software. The nominal aspect of the product is derived from the TTRS approach. The relationship between functional surfaces is determined by the graph tool [16]. Then, a kinematic analysis of the mechanism is performed by using the MECAmaster software. In CAD model, MGREs (Minimum Geometrical Reference Elements) are defined for each kinematic joint. At the end, the solid model can be deduced from the surface one. The proposed approach allows the definition of 


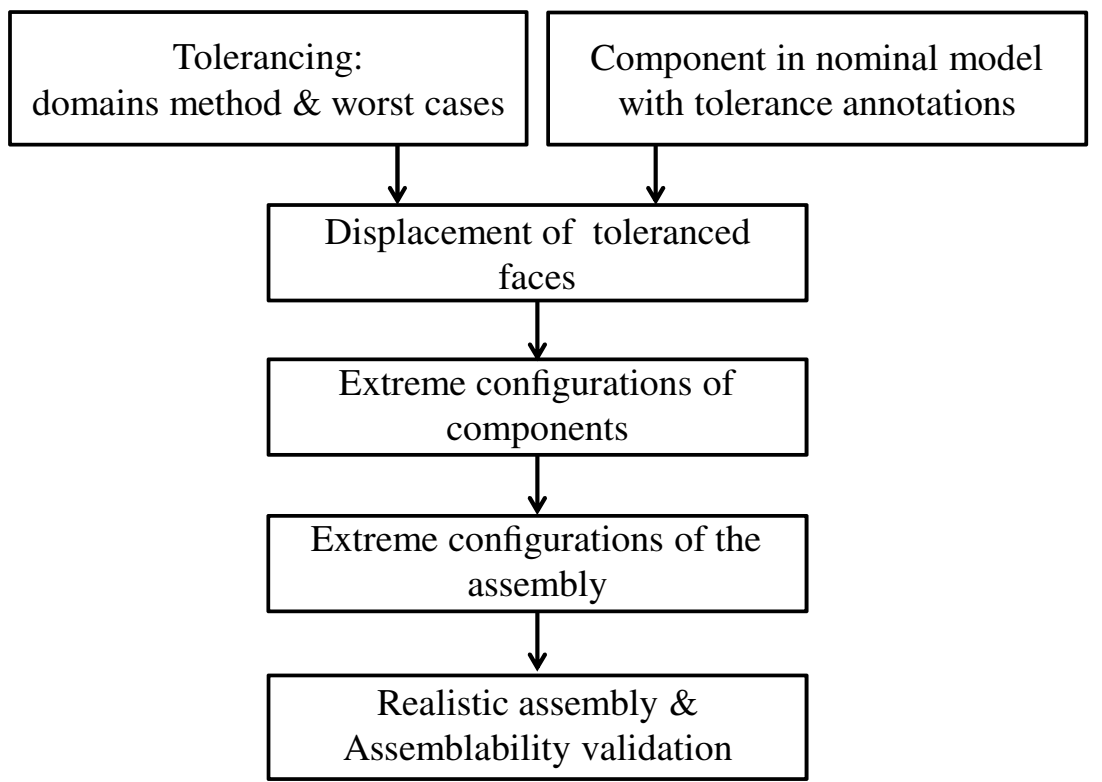

Fig. 2. Modelling of the realistic assembly.

a numerical model by functional surfaces incorporating tolerancing. Then, the geometric variations are respected in earliest step of PLM. Nevertheless, the model does not predict the impact of the tolerance choice on the system functioning.

Buysse et al. develop a model based on TTRS approach for the realistic simulation of assemblies [17]. Indeed, the determination of the MGRE leads to the representation of complex mechanical systems by a simple parametric model. Deviations of toleranced face points are represented by using polyhedral computation tools [9]. The impact of the tolerance choice and architecture on the assembly requirement is obtained. The comprehension of the polyhedral representation is a difficult task in the industry. The model does not predict the additional stresses due to tolerances.

Mandil et al. introduced a conceptual model to define a functional requirement during the product life cycle management [18]. The method is based on the parametric modelling of the TTRS approach and it determines the impact of thermo-mechanical stress deviations on the value of the functional condition. The deformations are calculated by using theoretical formulas (manually). The use of F.E. calculations is not possible because the method does not use a geometric modelling. The modelling has not been applied to the three-dimensional tolerancing chain and has not been automated.

Tsai et al. confirmed that the geometric and dimensional deviations due to tolerancing can be critical in a case of a component stack-up, especially when the component tolerances are pressed [2]. This hypothesis was confirmed by the results of F.E. calculations. In fact, the same boundary conditions were applied to two models to simulate the operation of the same mechanism: the first is a nominal model and the second is a model with defects. The results of F.E. calculations showed that deformations are different for both models. Thus, it is important to take into account the influence of tolerance accumulations on the mechanisms robustness during the design phase.

\section{Algorithm of realistic assemblies modelling}

The integration of tolerances in the geometric model allows obtaining realistic assemblies. These assemblies enable the possibility to evaluate tolerance impacts on assembly requirements and to predict assembly deformations. To obtain realistic assembly an algorithm is proposed (Fig. 2).

The methodology consists in determining worst case configurations of an assembly that are required and imposed by tolerances. These models are given by applying the worst case tolerancing $[9,19]$ to all toleranced faces of the components. In our study, the principle of tolerance independency according to ISO 8015 is considered. Mathematical formulations of tolerance zones are obtained by the domains method. Indeed, the SDTs are used to model the geometrical deviations. Then, the assumption of neglecting form defects relative to orientation and position defects is adopted. Hence, allowed extreme positions of faces are calculated. Thereafter, displacements parameters of faces, which are required by the tolerance, are deduced. Thus, realistic assemblies, which take into account the tolerances in CAD model, are obtained by rebuilding assemblies.

\section{A model taking into account tolerances in CAD model}

The consideration of the tolerances in CAD model is obtained by face displacements. Therefore, subalgorithms were developed to realize the desired 


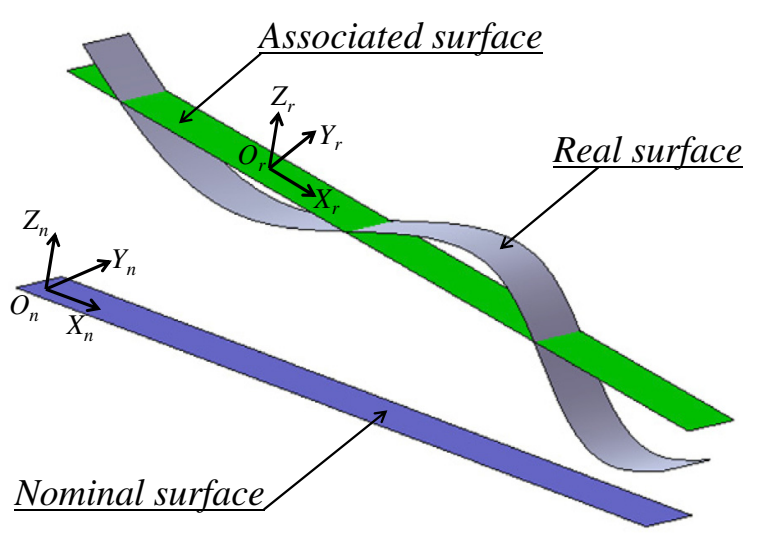

Fig. 3. Definition of the deviation torsor of a planar surface.

displacements of faces. Parameters of each displacement are calculated by using the domains method and a worst case approach. The deviation between nominal and realistic feature is determined by the SDT tool. Then, form deviations are neglected relative to those of orientation and position. The model is integrated under the CAD software "SolidWorks". The methodology to determine worst case faces depends on the type of the toleranced face. The face displacements and the identification of the tolerance type and the toleranced feature are automated.

\subsection{Displacements of a planar face}

In the case of planar surface, components of variations between the real coordinate system $R_{\mathrm{r}}\left(\mathrm{O}_{\mathrm{r}}, X_{\mathrm{r}}, Y_{\mathrm{r}}, Z_{\mathrm{r}}\right)$ (coordinate system of surface associated with real surface) and the nominal coordinate system $R_{\mathrm{n}}\left(\mathrm{O}_{\mathrm{n}}, X_{\mathrm{n}}, Y_{\mathrm{n}}, Z_{\mathrm{n}}\right)$ (coordinate system associated with nominal surface) are one translation and two rotations (Fig. 3). The deviation torsor $E p$ is defined on the face center O (Eq. (1)).

$$
\{E p\}_{\mathrm{o}}=\mathrm{o}\left\{\begin{array}{l}
r_{x} 0 \\
r_{y} 0 \\
0 t_{z}
\end{array}\right\}_{\left(\overrightarrow{\mathrm{n}_{\mathrm{n}}}, \overrightarrow{\mathrm{n}_{\mathrm{n}}}, \overrightarrow{z_{\mathrm{n}}}\right)}
$$

In order to illustrate the possible displacements of a planar face, a prismatic part is taken as an example (Fig. 4a). The toleranced planar face, defined by $a=50 \mathrm{~mm}$ and $b=40 \mathrm{~mm}$, is subjected to a positional tolerance $T_{1}=0.1 \mathrm{~mm}$. The determination of all configurations with worst case tolerancing for this surface requires the calculation of displacements of points I, J, K and L [9].

The positional tolerance requires that real surface must be included in a zone limited by two parallel plans distant of $T_{1}$. In addition, the median plane is parallel and distant from $\mathrm{L}$ to the reference face $\mathrm{A}$. The deviation torsor $E_{\mathrm{o}}\left\{r_{x}, r_{y}, 0,0,0, t_{z}\right\}$, expressed in coordinate system $(\mathrm{O}, \vec{x}, \vec{y}, \vec{z})$, is allotted to toleranced face. By neglecting form deviation relative to those of orientation and position, it is necessary and sufficient that four points I, $\mathrm{J}, \mathrm{K}$ and $\mathrm{L}$ (delimiting surface) are included in this zone. A point $\mathrm{M}$ is defined such as $M \in[\mathrm{I}, \mathrm{J}, \mathrm{K}, \mathrm{L}]$ and $\partial \vec{M}$ the vector displacement of the point $M$. The localization tolerance requires that: $-\frac{T_{l}}{2} \leq \partial \vec{M} \cdot \vec{z} \leq \frac{T_{l}}{2}$; such as

$$
\partial \vec{M}=\partial \vec{G}+\overrightarrow{M G} \wedge \partial \vec{\Omega}
$$

with $\partial \vec{\Omega}=\left(\begin{array}{c}r_{x} \\ r_{y} \\ 0\end{array}\right)$ Then, we obtain the relation (Eq. (2)).

$$
\left\{\begin{aligned}
-\frac{T_{1}}{2} & \leq t_{z}+\frac{a}{2} r_{y}+\frac{b}{2} r_{x} \leq \frac{T_{1}}{2} \\
-\frac{T_{1}}{2} & \leq t_{z}+\frac{a}{2} r_{y}-\frac{b}{2} r_{x} \leq \frac{T_{1}}{2} \\
-\frac{T_{1}}{2} & \leq t_{z}-\frac{a}{2} r_{y}+\frac{b}{2} r_{x} \leq \frac{T_{1}}{2} \\
-\frac{T_{1}}{2} & \leq t_{z}-\frac{a}{2} r_{y}-\frac{b}{2} r_{x} \leq \frac{T_{1}}{2}
\end{aligned}\right.
$$

Surface extreme configurations are obtained by determining extreme values of $t_{z}, r_{x}$ and $r_{y}$. Therefore, the following six worst cases are given:

- A rotation about the $\overrightarrow{o x}$ axis by an angle $T_{1} / b$ or $-T_{1} / b$.

- A rotation about the $\overrightarrow{o y}$ axis by an angle $T_{1} / a$ or $-T_{1} / a$.

- A translation along the $\overrightarrow{o z}$ axis by a value $T_{1} / 2$ or $-T_{1} / 2$.

A sub-algorithm is developed to determine the worst case configurations of the Planar Face with Quadratic Loop which is subjected to Positional Tolerance (Sub-algorithm PFQLPT) (Fig. 5). First, a local Cartesian coordinate system $(\mathrm{O}, \vec{x}, \vec{y}, \vec{z})$ is defined on the toleranced face $[\mathrm{I}, \mathrm{J}, \mathrm{K}, \mathrm{L}]$. The $\vec{z}$ axis is normal to the face and the $\vec{x}$ axis is along the $\overrightarrow{\mathrm{IJ}}$ axis $(\vec{x}=\overrightarrow{\mathrm{IJ}} /\|\overrightarrow{\mathrm{IJ}}\|)$. Besides, the displacement can be a rotation or a translation in the case of planar face. In fact, two translations along to the face normal by $T_{1} / 2$ and $-T_{1} / 2$ values are realized. In addition, $\vec{x}$ axis rotation and $\vec{z}$ axis rotation are performed.

Figure $4 \mathrm{~b}$ shows the rotation method of a planar face about the $\vec{x}$ axis (small median of rectangle) by an angle equal to $T_{1} / b$. The rebuilding of the geometrical model requires the updating of the faces influenced by the displacement of that toleranced one.

\subsection{Case of a cylindrical tolerance zone}

In the case of positional tolerance $(t)$ assigned to an axis $\mathrm{AB}$ of a cylindrical face (or a conical face), the tolerance zone is a cylinder with diameter $(t)$ (Fig. 6a). The three data (A, B and C) are supposed ideal faces. Deviation components between the real coordinate system $R_{\mathrm{r}}$ $\left(\mathrm{O}_{\mathrm{r}}, \overrightarrow{X_{\mathrm{r}}}, \overrightarrow{Y_{\mathrm{r}}}, \overrightarrow{Z_{\mathrm{r}}}\right)$ and the nominal coordinate system $R_{\mathrm{n}}$ $\left(\mathrm{O}_{\mathrm{n}}, \overrightarrow{X_{\mathrm{n}}}, \overrightarrow{Y_{\mathrm{n}}}, \overrightarrow{Z_{\mathrm{n}}}\right)$ are defined as follows.

Geometric deviations are represented by SDT. Then, form defects are neglected relative to orientation and position defects (Fig. 6b). Displacements of the two end 


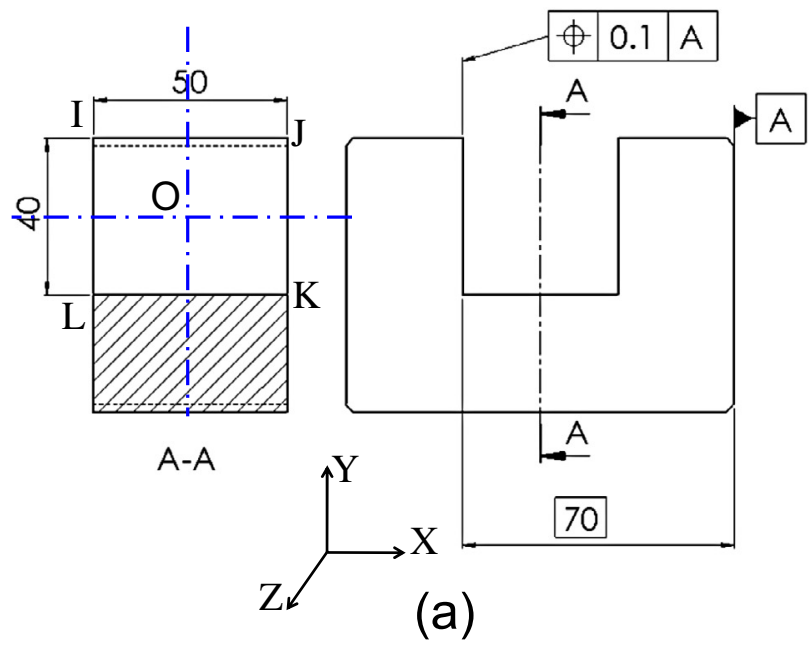

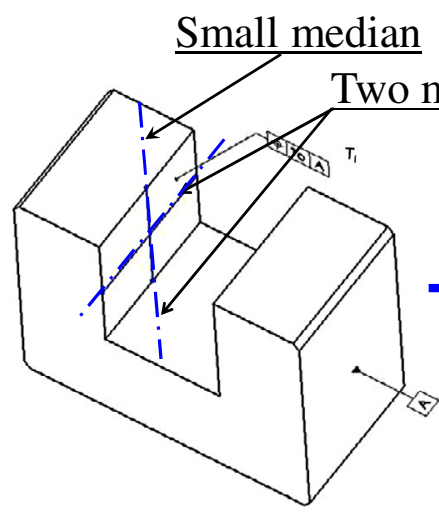

Nominal Model

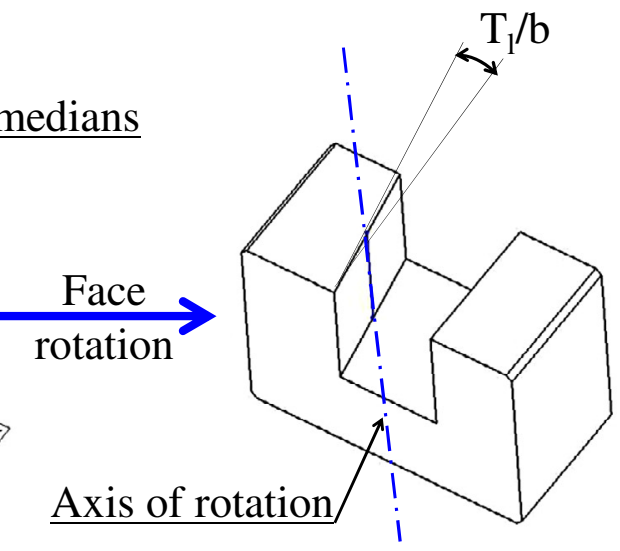

(b) Realistic Model

Fig. 4. (a) Planar face subjected to a positional tolerance. (b) Rotation of a planar face.

Component in nominal configuration: Planar face with geometric tolerance (annotation)
Tolerancing: worst cases + domains method

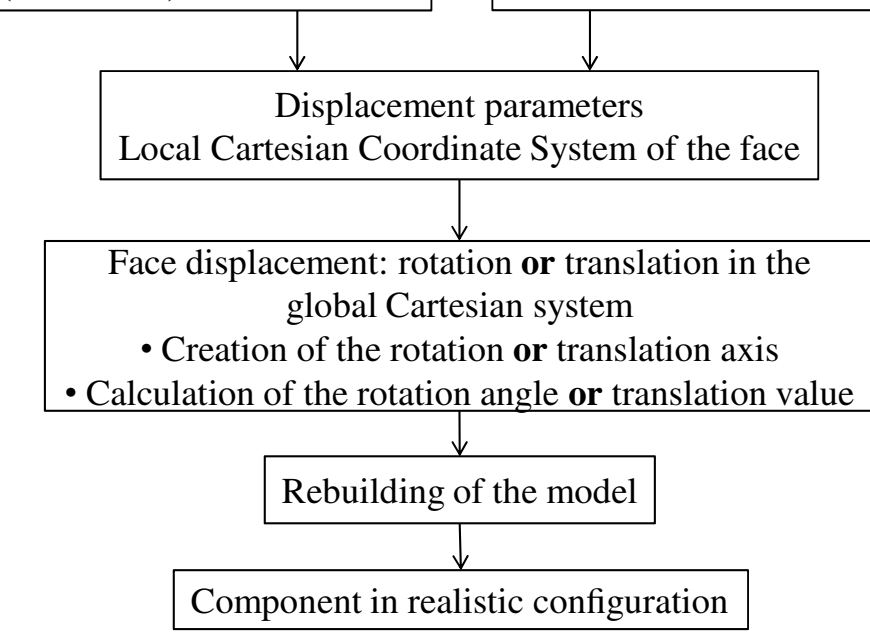

Fig. 5. Sub-Algorithm PFQLPT. 


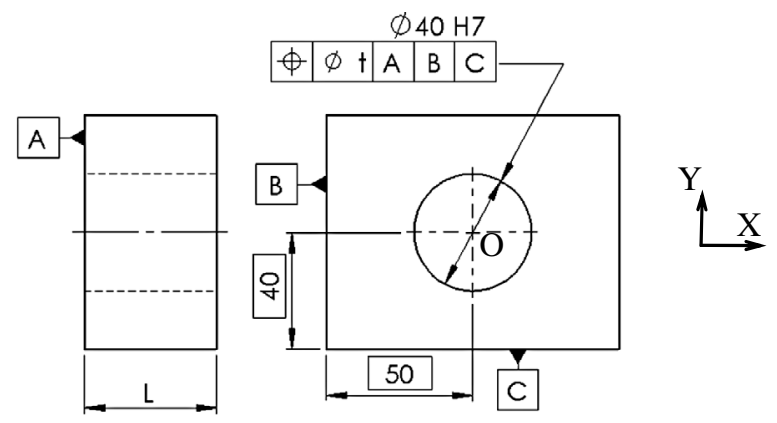

(a)

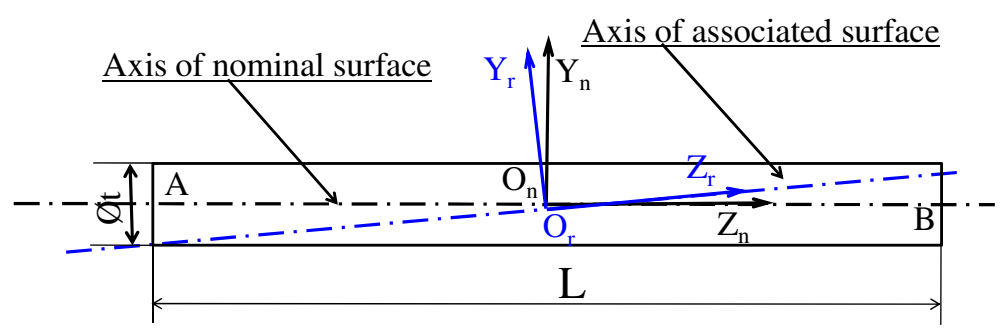

(b)

Fig. 6. (a) Positional tolerance allotted to cylinder axis. (b) Calculation of the deviation torsor of a cylindrical face.

points of the cylinder axis (A and B) must be lower than $t / 2$. Displacement vectors of the points $\mathrm{A}$ and $\mathrm{B}$ are deduced from displacement vector of the point $\mathrm{O}$. Then, the quadratic inequalities (Eq. (3)) are deduced in the plane $\left(\overrightarrow{X_{\mathrm{n}}}, \overrightarrow{Y_{\mathrm{n}}}\right)[20]$.

$$
\left\{\begin{array}{l}
\left(T_{x}-R_{y} L / 2\right)^{2}+\left(T_{y}+R_{x} L / 2\right)^{2} \leq(t / 2)^{2} \\
\left(T_{x}+R_{y} L / 2\right)^{2}+\left(T_{y}-R_{x} L / 2\right)^{2} \leq(t / 2)^{2}
\end{array}\right.
$$

$T_{x}$ and $T_{y}$ are two translation parameters and $R_{x}$ and $R_{y}$ are two rotation parameters.

To solve the equation (Eq. (3)) and to determine the realistic configurations of the axis, the discretization of the tolerance zone (virtual zone) is carried out (Fig. 7). This discretization can be performed by an angle $\alpha=2 k \pi / n ; k=1$ to $n$; where $n$ is the parameter of the discretization smoothness [9,20,21]. The parameter $n$ is chosen by the user according to the desired numbers of configurations and the accuracy of the results (Eq. (4)).

$$
\xi=t(1-\cos (\pi / n)) / 2
$$

$\xi$ is the deviation error of the discretization. The circular loop of toleranced zone is mathematically modelled by a set of edges. For the circular loop, the face normals at edge ends are defined in the coordinate system $(\mathrm{O}, \vec{X}, \vec{Y}, \vec{Z})$ by the following vector: $\overrightarrow{n_{k}}=(\cos (\alpha), \sin (\alpha), 0)$. Then, the tolerance constraint becomes:

$$
\left\{\begin{array}{l}
\overrightarrow{\delta A} \cdot \overrightarrow{n_{k}} \leq \frac{t}{2} \\
\overrightarrow{\delta B} \cdot \overrightarrow{n_{k}} \leq \frac{t}{2}
\end{array}\right.
$$

$\overrightarrow{\delta A}$ and $\overrightarrow{\delta B}$ are respectively the vector displacement of $\mathrm{A}$ and $\mathrm{B}$.

Thus, the relation (Eq. (5)) is deduced from equation (Eq. (3)).

$$
\left\{\begin{array}{l}
\left(T_{x}-\frac{L}{2} R_{y}\right) \cos \left(k \frac{2 \pi}{n}\right)+\left(T_{y}+\frac{L}{2} R_{x}\right) \sin \left(k \frac{2 \pi}{n}\right) \leq(t / 2) \\
\left(T_{x}+\frac{L}{2} R_{y}\right) \cos \left(k \frac{2 \pi}{n}\right)+\left(T_{y}-\frac{L}{2} R_{x}\right) \sin \left(k \frac{2 \pi}{n}\right) \leq(t / 2)
\end{array}\right.
$$

In the CAD model, the discretization is carried out by using the polar coordinate system [20]. Thus, the calculation of the coordinate transformation matrix is necessary to create axis $\left(\mathrm{O}, \overrightarrow{n_{k}}\right)$ in global coordinate system. The polar co-ordinates of the realistic axis ends are $(t / 2, \alpha)$. The displacement of the toleranced face implies the change of the adjacent faces geometry (Fig. 8).

Worst case configurations of the part (Fig. 6a) are calculated and modelled in CAD model according to the method detailed previously. Two cases are illustrated in Figure 9: a rotation of the cylindrical face with an angle $\operatorname{artg}(t / L)$ about the axis $\left(\mathrm{O}, \overrightarrow{n_{3}}\right)$ and a translation of the same face along the axis $\left(\mathrm{O}, \overrightarrow{n_{1}}\right)$ by a distance $(t / 2)$.

\subsection{Displacement of a planar face with non quadratic loop}

In the case of toleranced planar surface with non quadratic loop, the associated surface is the bounding polygonal one [21]. In fact, the surface loop is discretized by vertices. The $n$ vertices obtained by this discretization must remain inside the tolerance zone. Then, worst case 


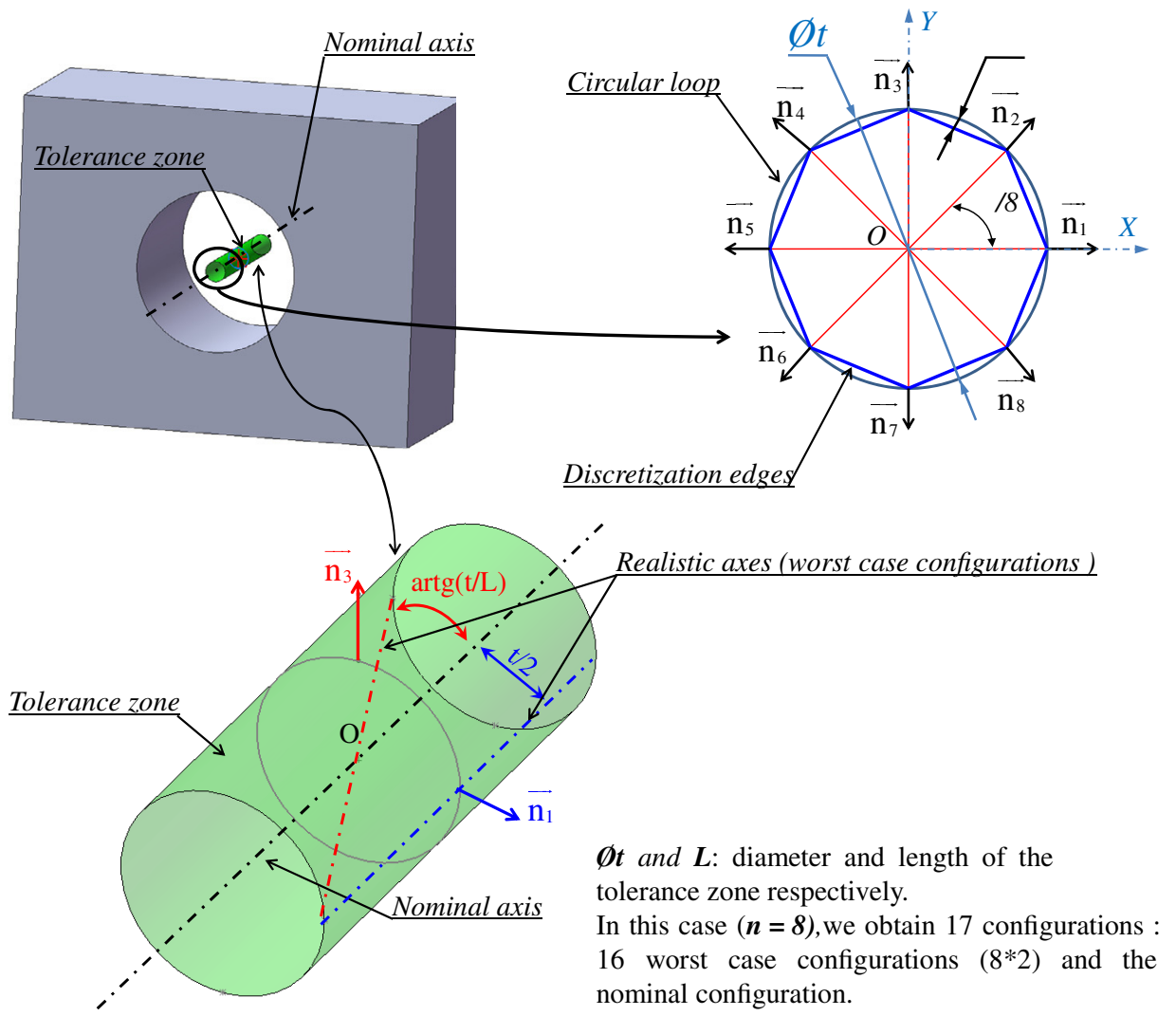

Fig. 7. A discretization of the tolerance zone (with $n=8$ ) to obtain worst case configurations of the axis.

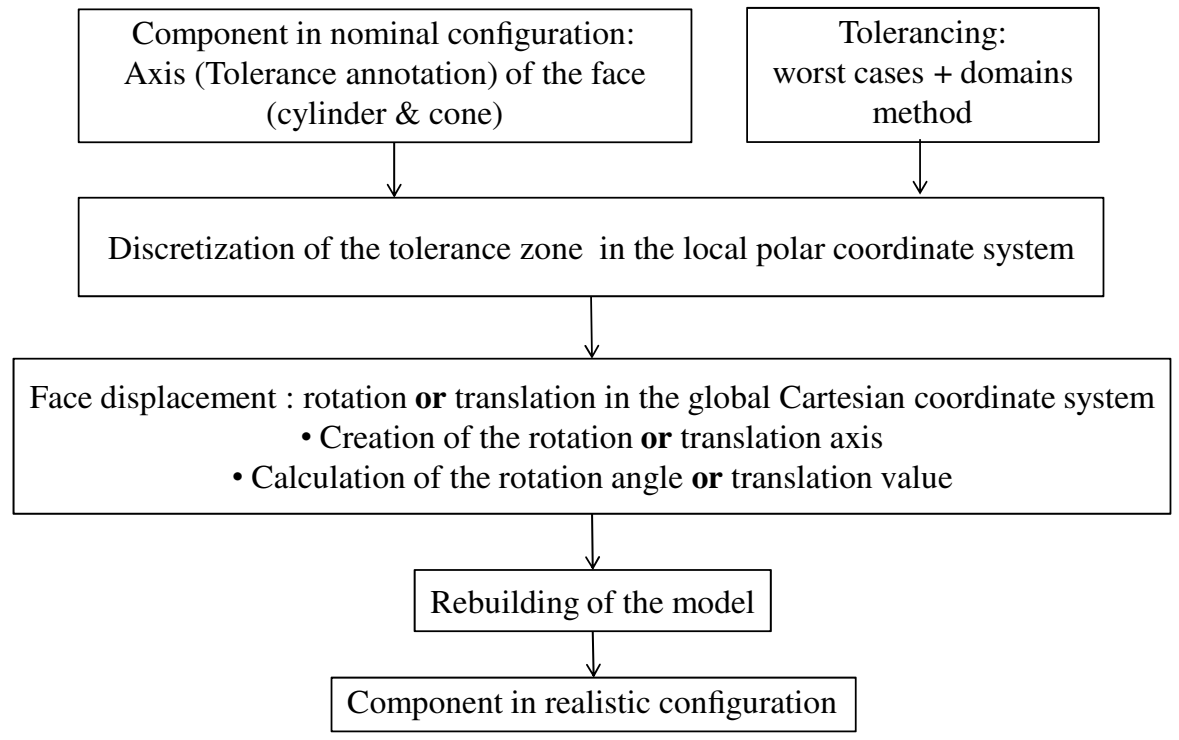

Fig. 8. Displacement of a face: case of the cylindrical tolerance zone.

displacements of the $n$ vertices are defined by $n$ inequalities. Indeed, the number of worst case configurations of the face is proportional to the number of discretization vertices. The number of configurations becomes very large, especially if the contour contains a circular portion.

The oriented bounding box (OBB) [22] allows enveloping the surface. The extremes OBB displacements along its eigenvectors are the extremes displacements of the corresponding surface. In the case of planar surface, the OBB is rectangular and planar. Then, the OBB is associated to toleranced surface. The identification of worst cases of toleranced surface is realized by using a method based on OBB (Fig. 10). In fact, worst case displacements of the toleranced face are deduced from worst case displacements of the corresponding OBB. 


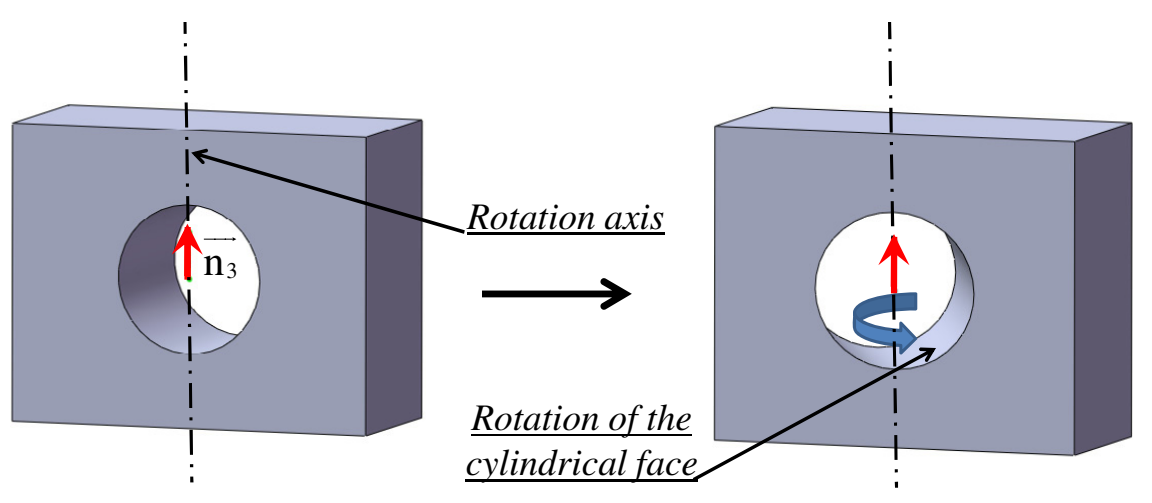

Nominal Part

Realistic Part

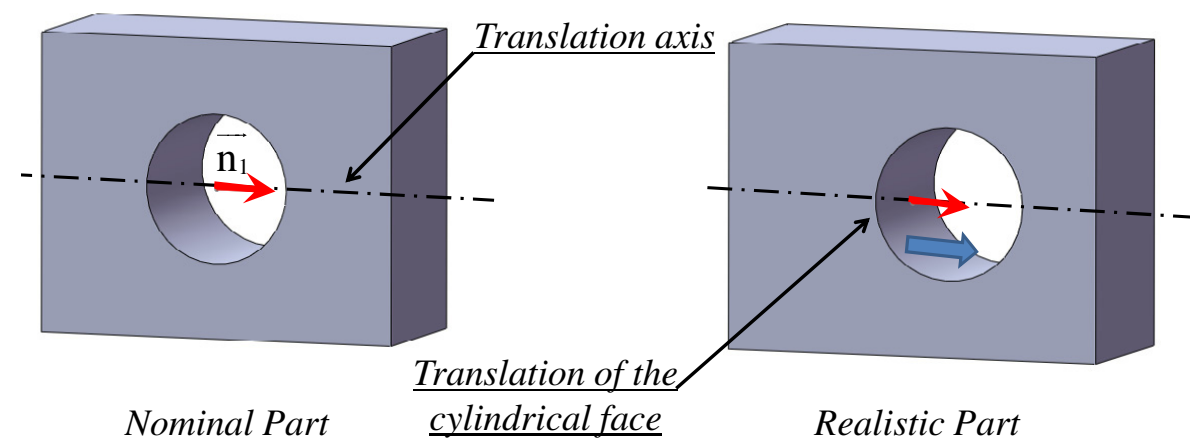

Fig. 9. Realistic modeling of a cylindrical face.

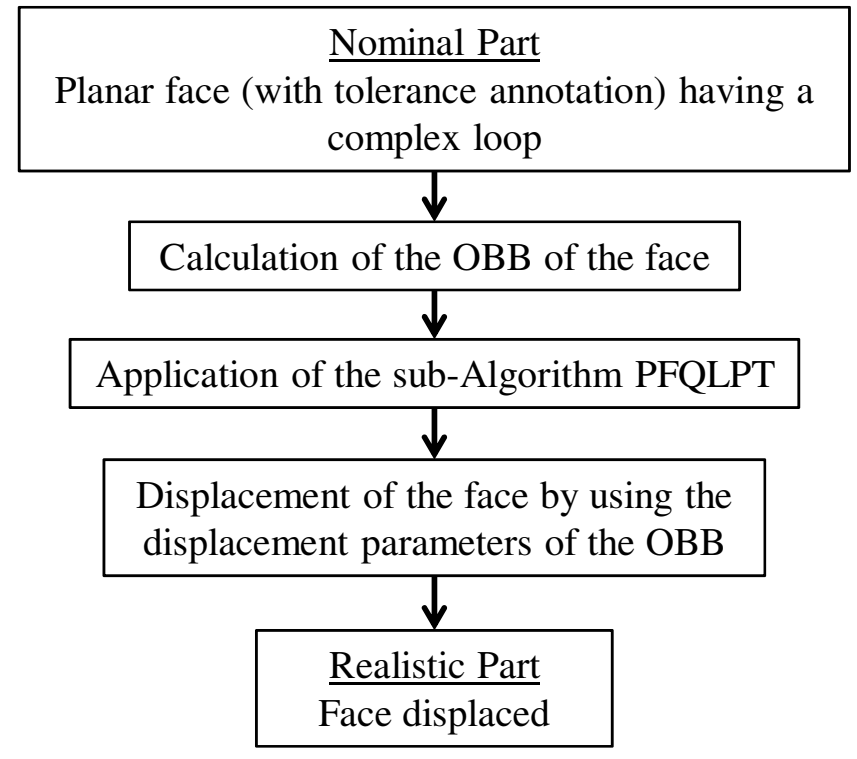

Fig. 10. Methodology of determination of face displacement parameters: case of planar face with complex loop.

Initially, the OBB of the toleranced face is calculated. The obtained OBB is a flattened parallelepiped (the box height is zero) and it has a four directing vertices (a rectangular loop). Hence, extreme displacements of driving vertices generate those of the face. Displacement settings of vertices are calculated by the previous algorithm used in the case of planar face with rectangular loop (sub-Algorithm PFQLPT in Fig. 5).

\section{Calculation of $O B B$}

The calculation of OBB of a surface is realized by using the following method.

In a CAD modelling, a surface is modelled by a tessellation [23]. Indeed, the tessellation is a triangulation approximating the face. Vertices coordinates of those representative triangles are extracted (Fig. 11). Then, the barycenter of each triangle is calculated by using the vectorial relation (Eq. (6)).

$$
C_{i}^{j}=\left(S_{1}^{i}+S_{2}^{i}+S_{3}^{i}\right) / 3
$$

In a Cartesian coordinate system of the CAD model, $C_{i}^{j}$ are coordinates of the barycenter of the triangle $(i)$ of the surface $(j)$ and $S_{k}^{i}$ are coordinates of a vertex of the triangle $(i)$ (each triangle has three vertices: $k=1$ to $3)$. Then, the barycenter of all vertices of the surface $(j)$ is determined by the application of the vectorial relation (Eq. (7)).

$$
C T^{j}=\frac{\sum_{i=1}^{N} C_{i}^{j}}{N}
$$

$C T^{j}$ is the coordinates vector of the barycenter of tessellation vertices of the surface $(j)$ and $N$ is the number of triangles in the surface $(j)$. A covariance matrix is 


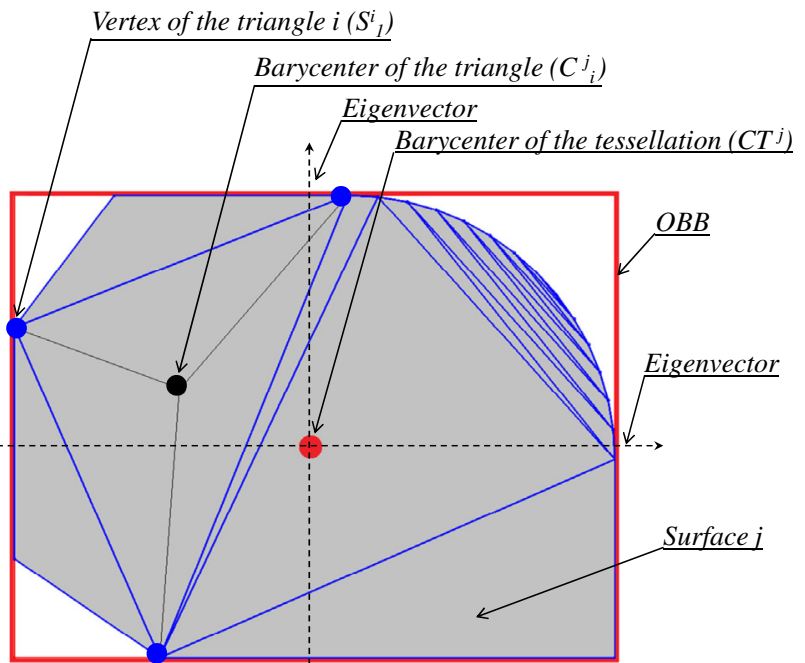

Fig. 11. Example of OBB.

a square matrix characterizing the interactions between variables [22]. Thereafter, the covariance matrix element $\operatorname{Cov}_{u, v}^{i}$ at row $u$ and column $v$ of each triangle $(i)$ is given by the vectorial equation (Eq. (8)).

$$
\begin{array}{r}
\operatorname{Cov}_{u, v}^{i}=\frac{1}{24}\left[\sum_{h=1}^{3} \sum_{k=1}^{3}\left(S_{h, u}^{i}-C T_{u}^{j}\right) *\left(S_{k, v}^{i}-C T_{v}^{j}\right)\right. \\
\left.+\sum_{k=1}^{3}\left(S_{k, u}^{i}-C T_{u}^{j}\right) *\left(S_{k, v}^{i}-C T_{v}^{j}\right)\right]
\end{array}
$$

Hence, the covariance matrix element $\operatorname{Cov} T_{u, v}^{j}$ ( $u$ row and $v$ column) of triangulated surface $(j)$ is given by the formula (Eq. (9)).

$$
\operatorname{Cov} T_{u, v}^{j}=\frac{\sum_{i=1}^{N}\left(A^{i} * \operatorname{Cov}_{u, v}^{i}\right)}{\sum_{i=1}^{N} A^{i}}
$$

$A^{i}$ is the area of the $i$ th triangle. The covariance matrix CovT, obtained by the equation (Eq. (9)), is symmetrical. In a coordinate system, the covariance matrix must be diagonal to obtain bounding box which will be close to the minimum bounding box: to obtain a minimum and oriented envelop including a vertex set. Then, there is a tridiagonal matrix $T$ and the orthogonal matrix $Q$ such as $T=Q^{T} \cdot \operatorname{Cov} T \cdot Q$. The diagonalization of the matrix $T$ by the Householder algorithm allows obtaining the eigenvectors [22]. Those obtained eigenvectors define the new orthonormal coordinate system and determine the OBB orientation. The projection of vertices coordinates on the new coordinate system allows determining the two extreme vertices in the box. Thus, the OBB of the surface is given. Figure 11 illustrates an example of OBB.
Determination of displacement parameters of the toleranced face

In order to illustrate the method of obtaining extreme configurations of a toleranced face by using an OBB, the example of Figure 12a is selected. A tolerance of localization $t_{0}=0.1 \mathrm{~mm}$ is required.

Initially, the OBB of the toleranced face is calculated (Fig. 12b). A tolerancing by using domains method is applied to the box (rectangular face). Indeed, the problem becomes similar to the case of a rectangular and planar face (sub-algorithm PFQLPT). Thus, the extreme configurations of the toleranced face will be included by the extreme configurations of OBB. Then, face displacement settings are those of the corresponding OBB. In Figure 12b, one possible worst case configuration is illustrated (the face displacement is amplified for easier viewing). The rotation axis and value are deduced from the OBB.

\section{Taking into consideration the MMC and the datum priority order in the CAD model}

The approach includes two main algorithms: the first algorithm, which is developed in previous works $[3,24]$, treats the dimensional tolerances. The second algorithm manipulates geometrical tolerances. The model respects the principle of independency. However, in the case of MMC or LMC (Maximum/Least Material Condition), a dependency exists between the geometric and dimensional tolerances. In addition, specification interpretation depends on the chosen datum reference. Then, this paper shows the ability of the developed model to respect those requirements: the two material conditions (MMC and LMC) and datum priority order. The validation will be illustrated by using the example in Figure 13 since the positional tolerance is the most used in industrial assembly. Initially, a first sub-algorithm allows defining the new realistic datum reference for repositioning the nominal axis of the hole. Then, a second sub-algorithm determines worst case configurations which are deduced from the MMC.

The position constraint of the hole (Fig. 13) mainly controls the position of the tolerance. In addition, it limits the form and orientation tolerances. The tolerance zone defined by the position tolerance is localized and oriented relatively to references by nominal dimensions (framed dimensions).

\subsection{Taking into consideration the datum reference order in the CAD model}

In datum reference frame, the choice of datum order depends on the part position in the assembly. In other words, it depends on a functional requirement.

The face $\mathrm{F}_{\mathrm{c}}$ (Fig. 13) is subjected to a localization constraint $T_{\mathrm{lC}}=0.5 \mathrm{~mm}$. Worst case configurations of $\mathrm{F}_{\mathrm{c}}$ 


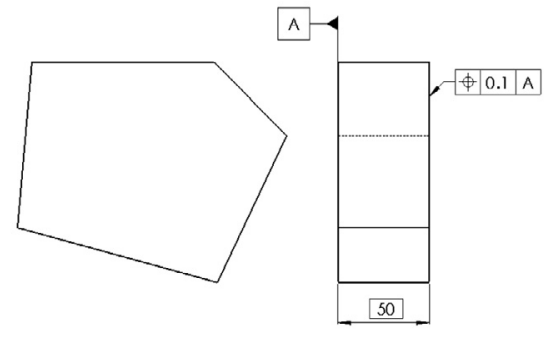

(a)

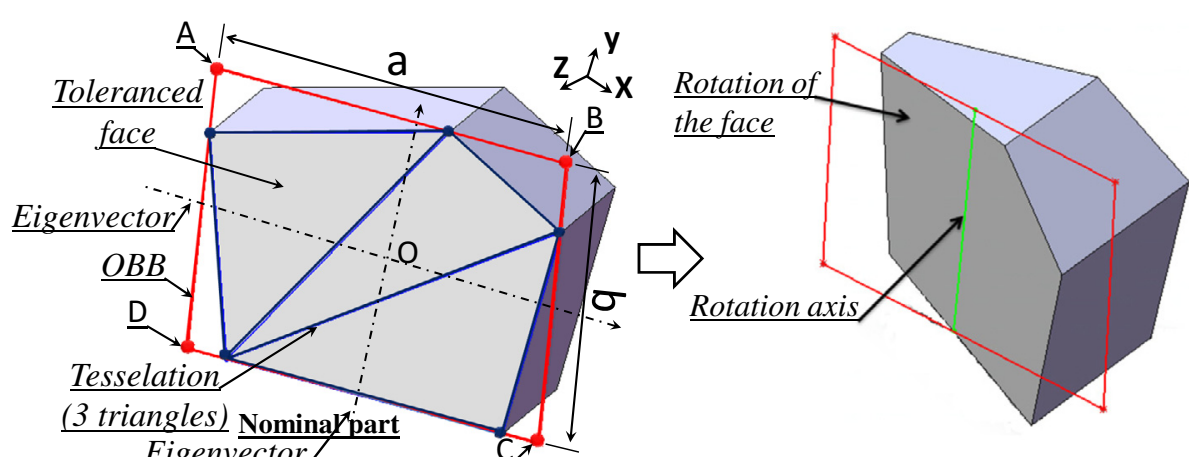

Eigenvector

Nominal part

(b)

$\underline{\text { Realistic part }}$

Fig. 12. (a) Part drawing. (b) Rotation of a toleranced face: case of planar face with complex loop.

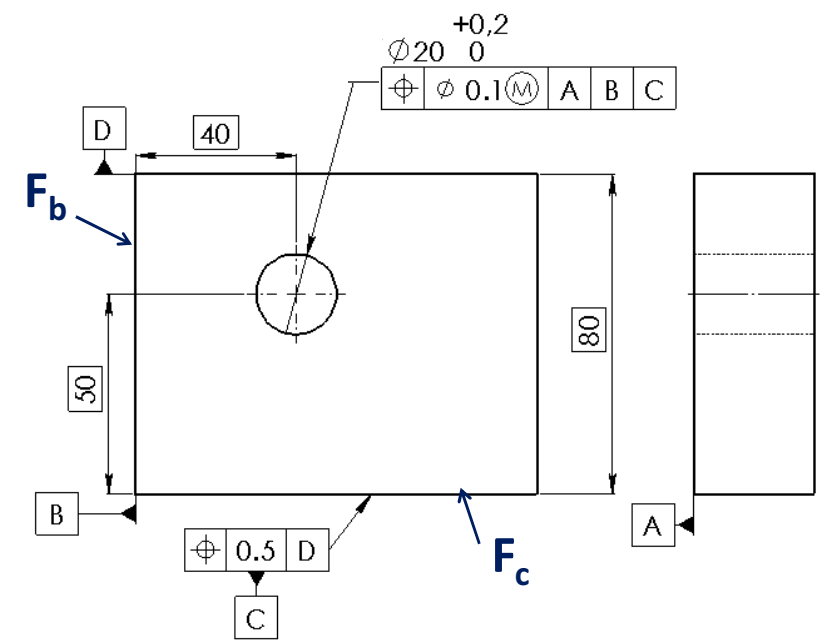

Fig. 13. Positional tolerance with a MMC and a tertiary datum reference.

are determined by using the model taking into account the geometrical tolerances. One of those configurations is shown in Figure $14 \mathrm{a}$ (rotation by $\alpha=0.23^{\circ}$ ). $\mathrm{F}_{\mathrm{b}}^{\prime}$ and $\mathrm{F}_{\mathrm{c}}^{\prime}$ (Fig. 14a) are the two non ideal faces obtained respectively from the two nominal faces $F_{b}$ and $F_{c}$. In this study, the face $\mathrm{F}_{\mathrm{b}}^{\prime}$ is preserved in its ideal configuration $\left(\mathrm{F}_{\mathrm{b}}=\mathrm{F}_{\mathrm{b}}^{\prime}\right)$ since it is not toleranced. $\mathrm{F}_{\mathrm{b}}^{\prime}$ and $\mathrm{F}_{\mathrm{c}}^{\prime}$ are not perpendicular.

For the datum reference frame $\left(\mathrm{S}_{\mathrm{a}}\right.$ : $\left.\mathrm{A}-\mathrm{B}-\mathrm{C}\right)$, the secondary datum $\mathrm{B}$ coincides with $\mathrm{F}_{\mathrm{b}}^{\prime}$ (since $\mathrm{B}$ respects the constraint of perpendicularity with the primary datum A in this configuration). Tertiary datum $\mathrm{C}$ is defined by $\mathrm{A}$ and B. C should be perpendicular to $\mathrm{A}$ as well as B. In the $\mathrm{CAD}$ model, the datum $\mathrm{C}$ does not coincide with the real face $\mathrm{F}_{\mathrm{c}}^{\prime}$ (Fig. 14a). At this step, the position of nominal axis from the datum $\mathrm{C}$ is equal to $50.4 \mathrm{~mm}$. Then, the axis position from datum $\mathrm{C}$ must be redefined to $50 \mathrm{~mm}$.

In digital mock-up, the circle center, from which the hole was created, is defined by two dimensions: $D 1=$ $40 \mathrm{~mm}$ relative to $\mathrm{F}_{\mathrm{b}}$ and $D 2=50 \mathrm{~mm}$ versus $\mathrm{F}_{\mathrm{c}}$ (made in the sketch on $\mathrm{F}_{\mathrm{a}}$ ). After the displacement of $\mathrm{F}_{\mathrm{c}}$, the center remains defined by the same dimension values $D 1$ and $D 2$. Indeed, in the feature manager design tree of the software, the sketch including the circle that appears before the displacement operation. Thus, the nominal position of the axis is not defined by the realistic datum reference. The position of nominal axis must be updated. Then, D2 is redefined relative to the new configuration of $\mathrm{F}_{\mathrm{c}}\left(\mathrm{F}_{\mathrm{b}}\right.$ is not changed). So, a new dimensional value $49.6 \mathrm{~mm}$ is assigned to D2 (Fig. 14b). The distance between nominal axis and the datum $\mathrm{C}$ becomes equal to $50 \mathrm{~mm}$.

A second datum reference frame $\left(\mathrm{S}_{\mathrm{b}}: \mathrm{A}-\mathrm{C}-\mathrm{B}\right)$ is chosen instead of $\mathrm{S}_{\mathrm{a}}$. The same worst case configuration of the part used previously is conserved. Secondary datum $\mathrm{C}$ coincides with the face $\mathrm{F}_{\mathrm{c}}^{\prime}$. The tertiary datum $\mathrm{B}$ is defined by $\mathrm{A}$ and $\mathrm{C}$. It is perpendicular to $\mathrm{A}$ as well as C. However, in the CAD model, B does not coincide with the real face $\mathrm{F}_{\mathrm{b}}^{\prime}$ (Fig. 15a). In order to reposition the axis center of the hole at a distance equal to $50 \mathrm{~mm}$ from the datum $\mathrm{C}$, the dimension $D 2$ of the sketch becomes equal to $49.84 \mathrm{~mm}$ (Fig. 15b). In addition, the dimension 


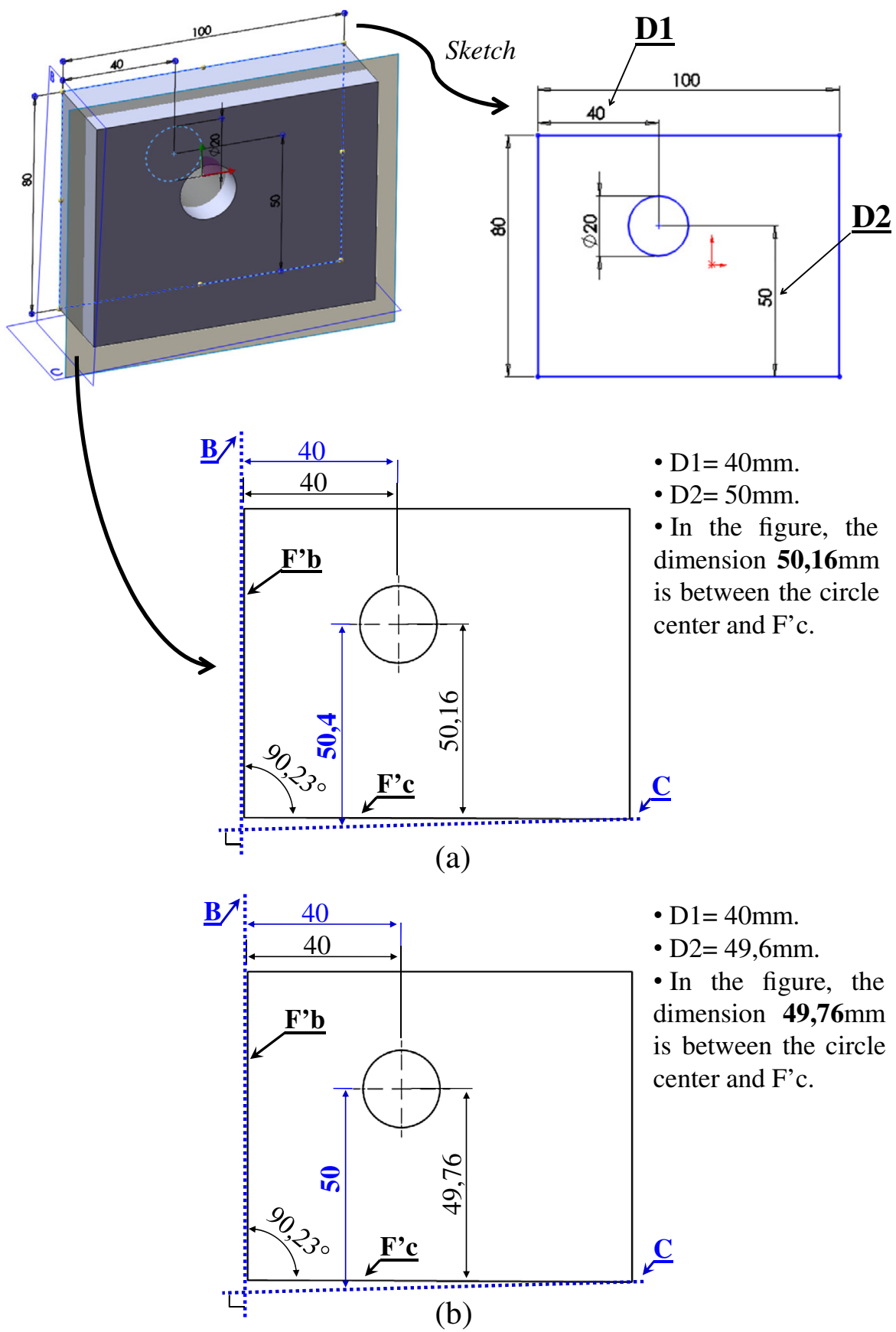

Fig. 14. (a) One realistic configuration of the part. (b) Redefining of the axis position ( $\mathrm{S}_{\mathrm{a}}$ : A-B-C).

$D 1$ of the sketch becomes equal to $39.88 \mathrm{~mm}$ to obtain a distance equal to $40 \mathrm{~mm}$ between the axis and B. Then, the cylinder axis is positioned by dimension values $40 \mathrm{~mm}$ and $50 \mathrm{~mm}$ relative to the data $\mathrm{B}$ and $\mathrm{C}$ respectively.

\section{General Algorithm for taking a datum reference order into consideration in the CAD model}

In the general case, a feature is constrained to a location tolerance defined relative to a tertiary datum reference (three data A1, A2 and A3 are respectively associated to three planar faces F1, F2 and F3). To take into account tolerances, which are attributed to F1, F2 and F3 (Fig. 16), the displacement of those faces to their corresponding realistic configurations is performed. Therefore, each realistic configuration of the part is defined by the three faces with defects $\mathrm{F}^{\prime} 1$, and $\mathrm{F}^{\prime} 2 \quad \mathrm{~F}^{\prime} 3$ (deduced respectively from nominal faces F1, F2 and F3). Thus, a realistic datum reference frame $\left(\mathrm{A}^{\prime} 1, \mathrm{~A}^{\prime} 2\right.$ and $\left.\mathrm{A}^{\prime} 3\right)$ can be deduced by using the sub-algorithm in Figure 16 . Indeed, the first datum coincides with realistic face $\mathrm{F}^{\prime} 1$ (since the form deviation is neglected in proposed model). 


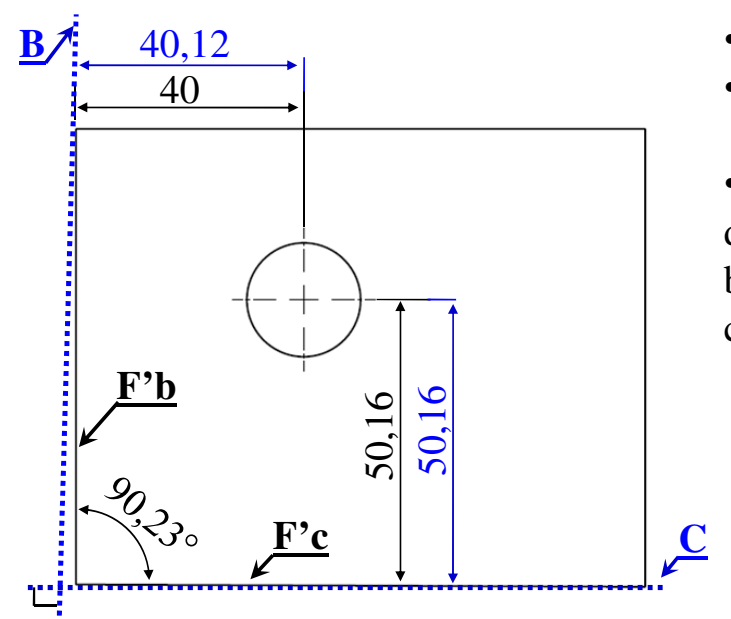

- $\mathrm{D} 1=40 \mathrm{~mm}$.

- D2 $=50 \mathrm{~mm}$.

- In the figure, the, dimension $\mathbf{4 0} \mathrm{mm}$ is between the circle center and F'b.

(a)

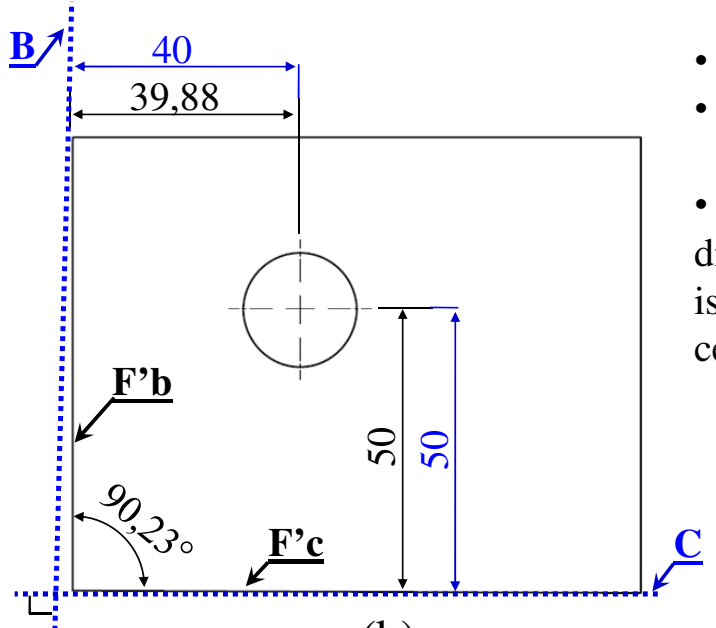

- $\mathrm{D} 1=39.88 \mathrm{~mm}$.

- D2 $=49.84 \mathrm{~mm}$.

- In the figure, the dimension $\mathbf{3 9 . 8 8} \mathrm{mm}$ is between the circle center and F'b.

(b)

Fig. 15. (a) One realistic configuration of the part. (b) Redefining of the axis position $\left(\mathrm{S}_{\mathrm{b}}\right.$ : A-C-B).

The secondary datum $\mathrm{A}^{\prime} 2$ is obtained with respect to the following two criteria: to be perpendicular to $\mathrm{A}^{\prime} 1$ and to be tangential free side of the material to $\mathrm{F}^{\prime} 2$. Tertiary datum $\mathrm{A}^{\prime} 3$ must be perpendicular to $\mathrm{A}^{\prime} 1$ and to $\mathrm{A}^{\prime} 2$. In addition, $\mathrm{A}^{\prime} 3$ must be tangential free side of the material to $\mathrm{F}^{\prime} 3$. Using this new and realistic datum reference frame, the toleranced feature (such as a hole axis) is repositioned to keep the constraints of initial and nominal dimensions (framed).

\subsection{Taking into consideration the MMC requirement in the CAD model}

Requirements of the MMC and LMC indicate interdependence between the size and the geometry of toleranced feature. The MMC constraint allows enlarging the tolerance range (bonus tolerance), ensuring mountability components and reducing the manufacturing cost.

The variation of the positional deviation $(t)$ of the part (Fig. 13) versus the diameter of the feature in nominal form $(D=25 \mathrm{~mm})$ can be represented in a dynamic diagram (Fig. 17). In general case, consider a hole with diameter $\phi D_{-t^{\prime} d}^{+t d}$ and an axis submitted to positional constrain $t_{0}$. The dimensional requirement defines a tolerance interval $I_{\mathrm{t}}$. The curve $\mathrm{C}$ of dynamic diagram in Figure 17 is defined by the following formula: $\mathrm{C}: t=a d+b$.

The following conditions must be respected: $\left\{t=t_{o} ; \quad\right.$ if $\quad d=D+t_{d}$

$\left\{=t_{o}+I_{\mathrm{t}} ; \quad\right.$ if $\quad d=D-t_{d}^{\prime}$.

Then $a=-\frac{I_{t}}{t_{d}+t_{d}^{\prime}} ; b=t_{o}+\frac{I_{t}}{2}\left(1+\frac{2 D+t_{d}-t_{d}^{\prime}}{t_{d}+t_{d}^{\prime}}\right)$.

To determine worst case configurations that are allowed by tolerances of the studied part, both requirements must be considered simultaneously. Indeed, each worst case value of dimensional tolerance $t_{k}^{d}\left(t_{k}^{d}=-t_{\mathrm{d}}^{\prime}\right.$ or $\left.t_{k}^{d}=t_{\mathrm{d}}\right)$ defines a positional tolerance zone $\varnothing t_{k}^{0}$. Then, the sub-algorithm is used to determine worst case configurations in the case of cylindrical tolerance zone. Finally, worst case configurations of the part (Fig. 13) are obtained while respecting the MMC requirement and the datum reference order. 


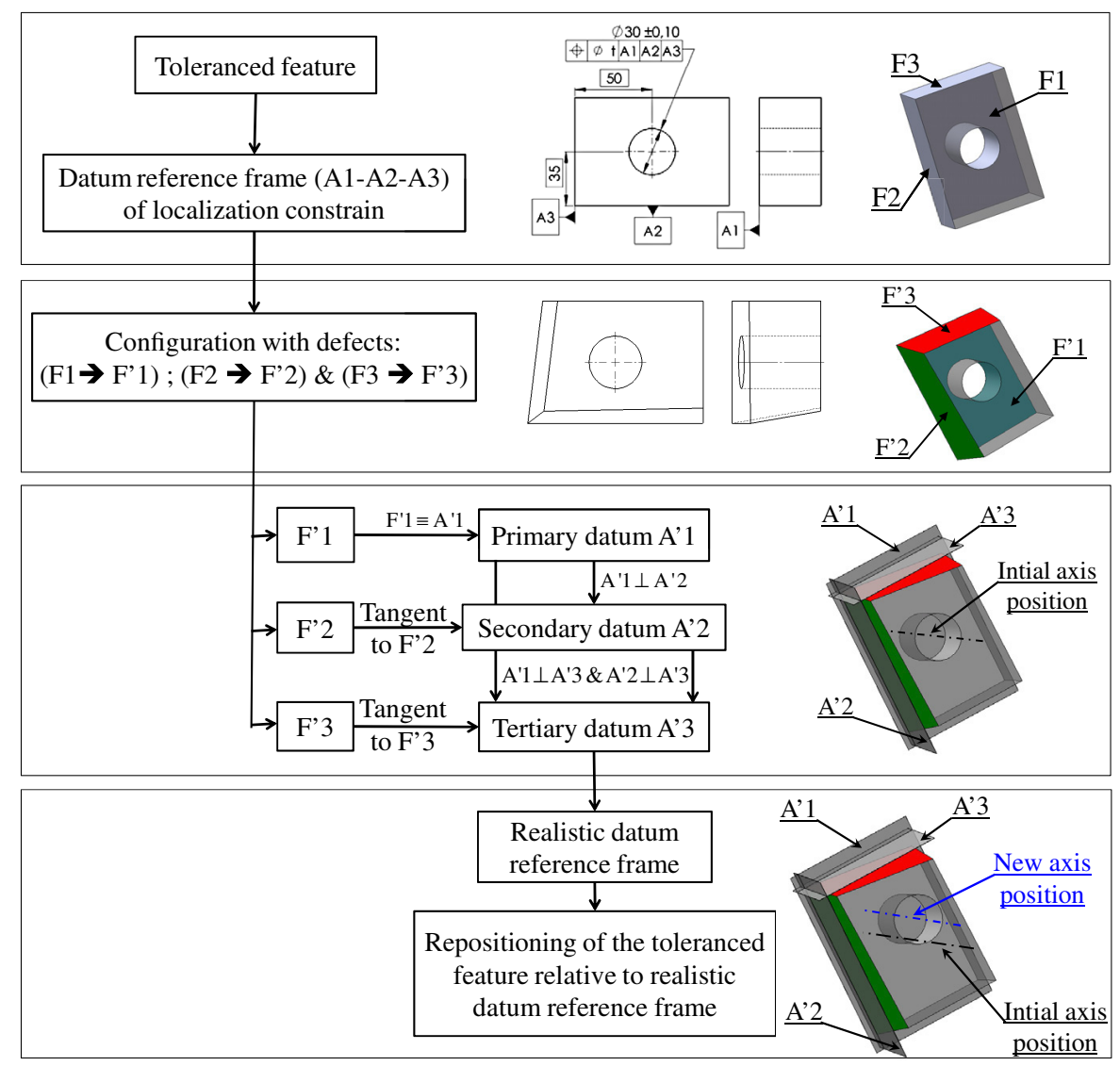

Fig. 16. Definition of realistic datum frame.

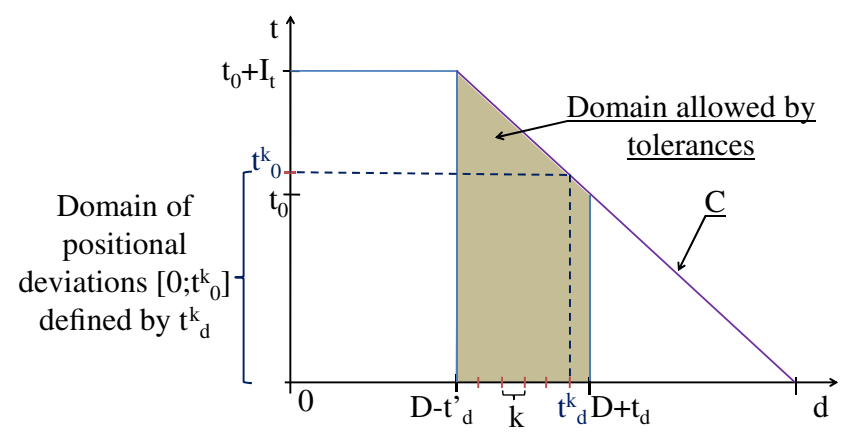

Fig. 17. The dynamic diagram.

\section{Realistic assembly application: inspection of functional requirement}

In the CAD environment, assembly components are represented by corresponding worst case configurations. Then, an update of CAD model is needed to obtain realistic assemblies. Thus, the visualization and the obtaining of tolerance impact on the assembly can be realized. Consequently, the prediction of the tolerances impact on the functional requirements is possible. The linear guide mechanism in Figure 18 is selected for illustration.
The principal function of this system is to realize a translation motion. To simplify the problem, only a minimum clearance $(J \min =1 \mathrm{~mm})$ is required in order to ensure the mechanism functioning. The geometrical and dimensional tolerances applied to the various components are illustrated in Figure 18 (only some tolerances are marked and the details are removed in the drawings to simplify the illustration). Then, worst case configurations of each component are determined (as indicated in the previous paragraphs). After obtaining the worst case configurations of assembly, the designer can detect the influence of selected tolerances on the functional requirement. The detection is realized by using the function for assembly analysis existing in CAD software (detection of collisions). Indeed, Figure 19 shows one of worst case configurations of the mechanism. In this configuration, the mechanism functioning is impossible. Thus, the choice of the tolerances values is not adequate.

\section{Research perspectives}

The proposed model uses the SDTs to represent the geometrical deviations. Thus, form defects are neglected relative to position and orientation errors. In fact, only form defects, that are systematically included on position errors, are considered. However, form specifications 

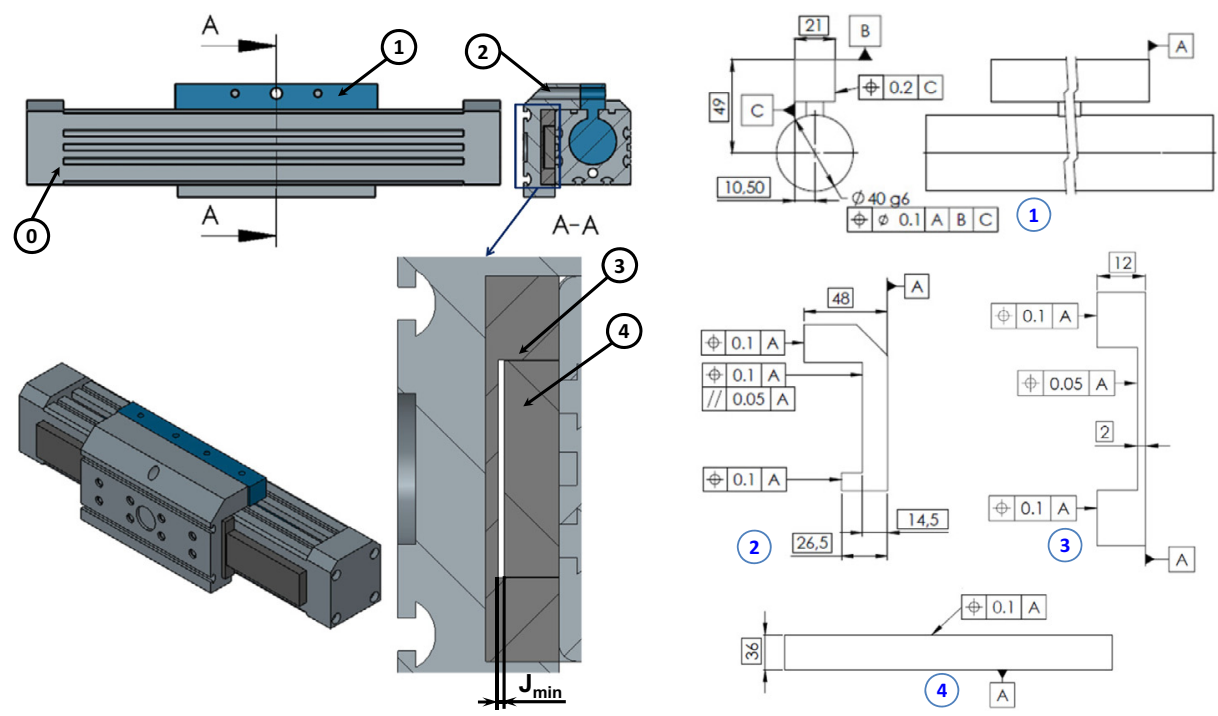

Fig. 18. Linear guide mechanism.
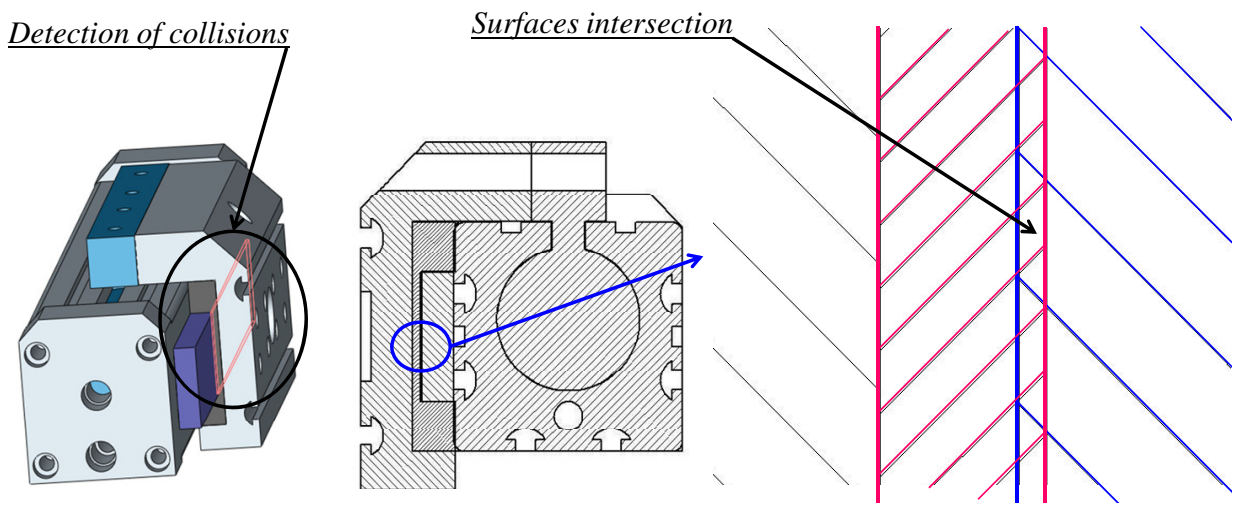

Fig. 19. Detection error due to tolerancing.

have effects on assembly requirements and can be incorporated on CAD model through another type of tolerancing approach which is a specific modelling of form defects [25]. In addition, the developed methodology is based on the SolidWorks API (Application Programming Interface). Thus, it depends on architecture of CAD software. To consider these two arguments, current research works, focuses on improving the proposed model. Initially, the components are meshed (Fig. 20). The feature defects deduced from tolerances are represented by the displacements of mesh nodes. Then, a CAD model is obtained by using the technique of reconstruction of CAD model from meshing $[26,27]$. Finally, realistic configurations of components lead to obtain the realistic assemblies.

In addition, the model proposed in this paper allows predicting tolerances impact on the assembly robustness. In fact, realistic models are used in a F.E. calculation which simulates the functional constraints of the studied mechanism. Deduced results are compared with those obtained by using the nominal model and applying same boundary conditions. Then, the impact of the tolerances stack-up on the F.E. results is predicted. In addition, the realistic models can be used in DMU to simulate many phases of PLM as dynamic calculation.

\section{Conclusion}

In this paper, a model is presented in order to obtain realistic assemblies. The model enables the tolerance analysis. The approach is based on tolerancing by the domains method and on the approach of worst cases. The realistic model is obtained by displacements of toleranced features to worst case configurations.

Sub-algorithms are developed to manipulate some particular cases. In fact, the tool of oriented bounding box allows circumventing the displacement problem of planar faces with complex loop. In addition, the MMC (or LMC) requirement and datum priority order are respected in the proposed model.

The difference between the numerical model and the real product is reduced by the proposed model. Then, in the digital Mock-up, the control of functional requirements and the optimization of assembly deformations are performed with realistic model. 


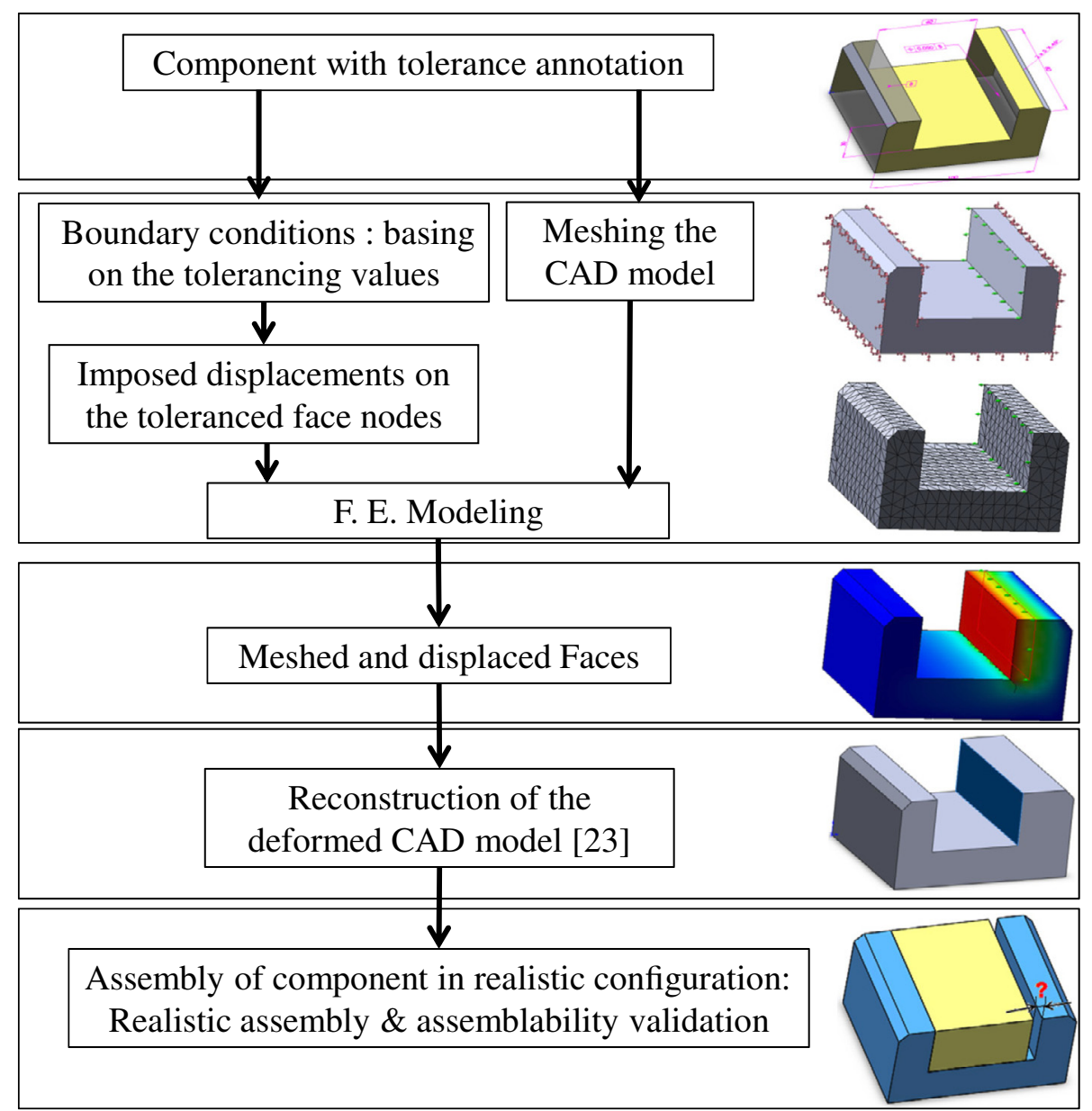

Fig. 20. Displacement of the toleranced faces by using the technique of CAD model rebuilding from meshing.

\section{References}

[1] G. Ameta, P. Hoffmann, Ontological model of tolerances for interoperability in product lifecycle, Ontological Model of Tolerances for Interoperability in Product Life Cycle, Chapt. 26, in: M. Giordano, L. Mathieu, F. Villeneuve (eds.), Geometric Variations within Product Lifecycle management (Selected Papers from the 11th CIRP International Conference on Computer-Aided Tolerancing, held at Université de Savoie, Annecy, France, 26-27 March 2009) ISTE, HERMES publishers, London, Uk, 2010, pp. 455-468

[2] J.C. Tsai, Stiffness variation of compliant devices due to geometric tolerancing, Proceedings of International Seminar on Computer-Aided Tolerancing 10th CIRP, Erlangen, Germany, 2007

[3] M. Tlija, B. Louhichi, A. BenAmara, Integration of tolerances in the mechanical product process, Proceedings of International conference on Innovative Methods in Product Design, Venice, Italy, 2011

[4] J. Dufaure, D. Teissandier, Geometric tolerancing from conceptual to detail design, Proceedings of CIRP Seminar on Computer-Aided Tolerancing, 2003, pp. 176-186

[5] B. Anselmetti, H. Louati, Generation of manufacturing tolerancing with ISO standards, Int. J. Mach. Tools Manuf. 45 (2005) 1124-1131
[6] A. Clement, A. Riviere, P. Serre, The TTRS: a common declarative model for relative positioning, tolerancing and assembly, International journal of CAD/CAM and computer graphics 11 (1996) 149-164

[7] A. Desrochers, W. Ghie, L. Laperriere, Application of a unified Jacobian-Torsor model for tolerance analysis, J. Comput. Inf. Sci. Eng. 3 (2003) 2-14

[8] F. Germain, M. Giordano, A new approach for threedimensional statistical tolerancing, Proceedings of CIRP Conference on Computer Aided Tolerancing, Erlanger, Germany, 2007

[9] J.Ph. Petit, S. Samper, Tolerancing analysis and functional requirement, Proceedings of the 5th International Conference on Integrating Design and Manufacturing in Mechanical Engineering, Bath, UK, 2004

[10] P. Bourdet, E. Ballot, Geometrical Behavior Laws for Computer-aided Tolerancing, Computer-aided Tolerancing, in: Chapman and Hill, F. Kimura (ed.), Proceedings of the 4th CIRP Design Seminar The University of Tokyo, Tokyo, Japan, 1995, pp. 119-131

[11] S. Tichadou, O. Legoff, J.-Y. Hascoët, 3D Geometrical Simulation of Manufacturing, Compared approaches between integrated CAD/CAM system and small displacement torsor model, Advances in Integrated Design and Manufacturing in Mechanical Engineering, Kluwer Academic Press, 2005, pp. 446-456. 
[12] F. Vignat, F. Villeneuve, A numerical approach for 3D manufacturing tolerances synthesis, Proceedings of CIRP Conference on Computer Aided Tolerancing, Erlangen, Germany, 2007

[13] R. Benea, G. Cloutier, C. Fortin, Process plan validation including process deviations and machinetool error, Geometric Product Specification and Verification: Integration of Functionality, Kluwer Academic Publishers, Netherlands, 2003, pp. 197-206

[14] M. Chang, D.C. Gossard, Modeling the assembly of compliant non-ideal parts, Comput. Aided Des. 29 (1997) 701-708

[15] A. Ballu, H. Falgarone, N. Chevassus, L. Mathieu, A new design method based on functions and tolerance specifications for product modelling, CIRP annals Manufactoring Technology 55 (2006) 139-142

[16] A. Ballu, L. Mathieu, Choice of functional specifications using graphs within the framework of education, Proceedings of 6th CIRP Seminar on Computer Aided Tolerancing, Enschede (The Netherlands), Kluwer Academic Publishers, 1999, pp. 197-206

[17] D. Buysse, M. Socoliuc, A. Rivière, A new specification model for realistic assemblies simulation, Proceedings of International Seminar on Computer-Aided Tolerancing 10th CIRP, Erlangen, Germany, 2007

[18] G. Mandil, A. Desrochers, A. Rivière, Framework for the monitoring of functional requirements along the product life cycle, Proceedings of CPI'2009, Fes, Morocco, 2009

[19] M. Pillet, P-A. Adragn, F. Germain, Inertial Tolerancing: The Sorting Problem, Journal of Machine Engineering: Manufacturing Accuracy Increasing Problems, Optimization 6 (2006) 95-102

[20] M. Giordano, S. Samper, J.P. Petit, Tolerance analysis and synthesis by means of deviation domains, axi-symmetric cases, in: J.K. Davidson (ed.), Models for Computer Aided Tolerancing in Design and Manufacturing 2007, Proceedings of the 9th CIRP International Seminar on Computer-Aided Tolerancing, held at Arizona State University, Tempe, Arizona, USA, 2005, pp. 85-94

[21] J.P. Petit, Spécification géométrique des produits: méthode d'analyse de tolérances. Application en conception assistée par ordinateur, Mechanical Ph.D. Thesis, Engineering High School of Annecy, 2004

[22] M. Lahanas, T. Kemmerer, N. Milickovic, K. Karouzakis, D. Baltas, N. Zamboglou, Optimized bounding boxes for three-dimensional treatment planning in brachytherapy, Med. Phys. J. 27 (2000) 2333-2342

[23] E. Béchet, J.C. Cuillière, F. Trochu, Generation of a Finite Element Mesh from Stereolithography (STL) Files, Comput. Aided Des. 34 (2002) 1-17

[24] B. Louhichi, M. Tlija, A. BenAmara, V. François, Reconstruction d'un modèle CAO à partir d'un maillage : application dans le cas de grands déplacements, Mécanique \& Industries 10 (2009) 477-486

[25] S. Samper, P.-A. Adragna, H. Favreliere, M. Pillet, Modeling of 2D and 3D Assemblies Taking Into Account Form Errors of Plane Surfaces, J. Comput. Inf. Sci. Eng. 9 (2009) 041005

[26] B. Louhichi, A. Benamara, V. Francois, Intégration $\mathrm{CAO} / \mathrm{Calcul}$ par reconstruction des modèles $\mathrm{CAO}$ à partir des résultats de calcul, Revue internationale d'ingénierie numérique 1 (2005) 9-26

[27] B. Louhichi, Intégration $\mathrm{CAO} / \mathrm{Calcul}$ par reconstruction du modèle $\mathrm{CAO}$ à partir des résultats éléments finis, Ph.D. Thesis, National Engineering School of Monastir, Tunisia, 2008 